\title{
The Factors Affecting Bone Density in Cirrhosis
}

\author{
Asghar Hajiabbasi ${ }^{1}$; Afshin Shafaghi ${ }^{2, *}$; Haniyeh Sadat Fayazi ${ }^{3}$; Irandokht Shenavar \\ Masooleh ${ }^{1}$; Mohammad Hassan Hedayati Emami ${ }^{1}$; Pooneh Ghavidel Parsa ${ }^{1}$; Alireza Amir \\ Maafi $^{4}$
}

${ }_{1}^{1}$ Guilan Rheumatology Research Center, Razi Hospital, School of Medicine, Guilan University of Medical Sciences, Rasht, IR Iran ${ }_{3}^{2}$ Gasteroenterology and Liver Disease Research Center, School of Medicine, Guilan University of Medical Sciences, Rasht, IR Iran

${ }_{4}^{3}$ Razi Hospital, School of Medicine, Guilan University of Medical Sciences, Rasht, IR Iran

${ }^{4}$ Student Research Center, Guilan University of Medical Sciences, Rasht, IR Iran

${ }^{*}$ Corresponding Author: Afshin Shafaghi, Gasteroenterology and Liver Disease Research Center, School of Medicine, Guilan University of Medical Sciences, Rasht, IR Iran Tel:+98-9111368274, Fax:+98-1315530169, E-mail: drafshinshafaghi@gmail.com

Received: January 10, 2015; Revised: February 17, 2015; Accepted: March 15, 2015

\begin{abstract}
Background: Bone loss is common in cirrhosis. However, the prevalence of osteopenia and osteoporosis has been heterogeneous in different reports. Reduction in bone formation with or without increase in bone resorption appears to be responsible for bone loss in these patients.

Objectives: We aimed to investigate bone loss in patients with cirrhosis at different anatomical sites and key factors that might affect it. Patients and Methods: In this cross-sectional study, 97 patients with cirrhosis who were referred to Razi Hospital, Rasht, Iran, from 2008 to 2010, were studied. Cirrhosis was diagnosed using biopsy and/or clinical and paraclinical findings. Bone mineral densitometry was done in L2 through L4 lumbar spine (LS) and femoral neck (FN), using dual-energy X-ray absorptiometry (DEXA) (QDR 1000, Hologic DEXA Inc, Waltham, Massachusetts, the United States). Statistical analysis was performed using SPSS 18. A P value $<0.05$ was considered statistically significant.

Results: A total of 97 patients with cirrhosis ( $55.7 \%$ male) and the mean age of $51 \pm 13$ years and median body mass index (BMI) of $22.7 \mathrm{~kg} /$ $\mathrm{m}^{2}$ were recruited over a two-year period. Etiologies of cirrhosis were hepatitis $\mathrm{C}(40.2 \%)$, hepatitis $\mathrm{B}(26.8 \%)$, cryptogenic (21.6\%), and other causes (11.4\%). Child A, B, and C, were seen in 16.5\%, $47.4 \%$, and $36.1 \%$ of patients, respectively. The DEXA results were abnormal in $78.4 \%$ of our participants (osteopenia, 45.4\%; osteoporosis, 33\%). BMI and calculated glomerular filtration rate (GFRc) had moderate positive and Child score had moderate negative significant correlation with $\mathrm{T}$ score in both anatomical sites. There was no significant association between abnormal DEXA and the causes of cirrhosis. The univariate analysis showed that the risk of abnormal results in DEXA was significantly higher in those with low BMI, current smoking, higher Child score, and low GFRc; however, in multivariate analysis, the abnormal results were more frequent in those with lower vitamin D, higher Child score, and less GFRc.

Conclusions: Abnormal DEXA was highly prevalent among patients with cirrhosis. The risk of this finding was increased by lower vitamin D levels, advanced disease, and impaired renal function.
\end{abstract}

Keywords: Bone Density; Osteoporosis; Liver Cirrhosis

\section{Background}

Bone mass at a specific time of life is determined by two factors: peak bone mass accumulated throughout growth, and consolidation and amount of bone loss that will occur thereafter. Peak bone mass, which is mainly determined genetically, can also be affected by environmental factors and lifestyle (1). The amount of bone loss during lifetime is determined by sex, age, body mass index (BMI), nutrition (e.g. the amount of dairy, salt, and protein), alcohol consumption, smoking, physical activity, some medications, and some diseases such as cirrhosis $(2,3)$. In patients with cirrhosis, pathogenesis of bone mass loss is still unknown (4), and most of our knowledge has been attained from studies on primary biliary cirrhosis (5). In cirrhosis, reduction in bone formation appears to be responsible for decrease in bone density although bone resorption might also have some roles (4). However, it has been shown that there is no significant difference in the T score of the lumbar spine (LS) or femur neck (FN) in patients with cirrhosis in comparison to the same scores in the normal individuals (6). In another study, there was lower bone mineral density (BMD) in patients with cirrhosis compared with that in normal controls (7). In addition, some studies have demonstrated that in patients with cirrhosis, the bone fracture is more prevalent in comparison with the general population, even after adjusting the confounding variables such as sex, presence of cholestasis, and high alcohol consumption (4, 8 ). The risk of fracture in cirrhosis, which is partly due to decreased BMD, increases by two-fold in comparison with the general population (4). Among existing methods for

Copyright (C) 2015, Kowsar Corp. This is an open-access article distributed under the terms of the Creative Commons Attribution-NonCommercial 4.0 International License (http://creativecommons.org/licenses/by-nc/4.0/) which permits copy and redistribute the material just in noncommercial usages, provided the original work is properly cited. 
BMD measurement, dual-energy X-ray absorptiometry (DEXA) is considered as the only practical method, which is clinically feasible (9). Improvement in the treatment of cirrhosis has increased the life expectancy of patients; therefore, special attention should be paid to their quality of life (QOL), which can be influenced by fracture.

\section{Objectives}

In this exploratory study, our aims were to assess bone loss in cirrhosis to determine key factors that can influence it.

\section{Patients and Methods}

This cross-sectional study was conducted during 20082010. Totally 97 consecutive patients with cirrhosis were studied who were referred to Razi Hospital, Rasht, Iran. This hospital is an educational and therapeutic referral center for patients with diseases related to all internal medicine fields. The exclusion criteria were as follows: pregnancy, disorders that might lower the accuracy of DEXA, weight $>120 \mathrm{~kg}$, past history of prolonged immobilization, disorders affecting bone metabolism (e.g. thyroid diseases, parathyroid diseases, diabetes, end-stage renal disease, rheumatoid arthritis, ankylosing spondylitis, inherited disorders of connective tissue, sarcoidosis, amyloidosis, hemophilia, thalassemia, hemochromatosis, and malignancies), and drugs affecting bone metabolism (e.g. supplements containing calcium and/ or vitamin D, cyclosporin, calcitonin, bisphosphonates, lithium, aluminum hydroxide, levothyroxine, corticosteroids, GnRH agonists, anticoagulants, anticonvulsant, antimetabolites, and cytotoxics). Written informed consent was obtained from all subjects after the approval of the study protocol by the Ethics Committee of Guilan University of Medical Sciences. Cirrhosis was diagnosed using biopsy, paraclinical results (e.g. prolonged prothrombin time, hypoalbuminemia, or increased bilirubin level), and clinical findings including signs and symptoms of cirrhosis, i.e. chronic stigmata of this disease, esophageal varices in endoscopy, or signs of liver cirrhosis in abdominal ultrasonography. Blood samples were also collected in the morning following a 12-hour overnight fast and were tested for calcium (Ca), phosphorus (P), alkaline phosphatase (ALP), blood urea nitrogen (BUN), creatinine (Cr), fasting blood sugar (FBS) (using auto-analyzer/HITA$\mathrm{CHI} 717$ ), and parathyroid hormone (PTH) levels as well as 25-hydroxyvitamin D [25(OH) vitamin D] levels (using ELISA/Stat Fax 3500). Among existing methods for BMD measurement, DEXA is considered as the only practical method, which is clinically feasible (9). Bone densitometry was done in LS (L2-L4) and FN, using DEXA (QDR1000, Hologic, DEXA Inc, Waltham, Massachusetts, the United States). The T score and its definition about osteopenia and osteoporosis were used in our analysis because the mean age of our patients was higher than 50 years (5). In the present study, osteopenia and osteoporosis in an anatomical site were defined as T score $\leq-1$ to $\geq-2.5$ and T score $<-2.5$, respectively (3). Diagnosis of osteoporosis was defined as existence of at least one anatomical site (LS or FN) with T score $<-2.5$. If DEXA-based diagnosis of patients could not be considered as normal bone density or osteoporosis, we categorized the patient as having osteopenia.

The weight of patients was measured after controlling ascites by one calibrated scale and all clinical assessment was performed by a trained assessor. We used Child-Pugh classification for clinical staging of cirrhosis. This reliable staging method has a scoring system of 5 through 15: score $\leq 6$, Child-Pugh group A; score 7 through 9, ChildPugh group B; and score 10 through 15, Child-Pugh group C (10). Glomerular filtration rate (GFR) was calculated by Cockcroft-Gault formula.Statistical analysis was performed using the SPSS 18 (SPSS Inc, Chicago, Illinois, the United States). Normal distribution of continuous variable was determined by Shapiro-Wilks' test. Correlation test, nonparametric two-related samples t test, MannWhitney U test, and logistic regression were also used. P value $<0.05$ was considered as statistically significant, but in multiple comparisons (Mann-Whitney U test), the significance was corrected to $\leq 0.01$.

\section{Results}

Ninety-seven patients with mean age $51 \pm 13$ years (range, 14 - 82 years) were studied in this research. Their basic characteristics are shown in Table 1 . About $80.2 \%$ of them had vitamin D deficiency ( $\leq 30 \mathrm{ng} / \mathrm{mL}$ ). The median BMI was $22.7 \mathrm{~kg} / \mathrm{m} 2$ (interquartile range $[\mathrm{IQR}]=2.1$ ), and we found that $78.4 \%$ (95\% CI, 70.2\% - 86.6\%) of the patients with cirrhosis had abnormal results in DEXA, $45.4 \%$ had osteopenia (38.6\% at LS, 6.9\% at FN, and 54.5\% at both sites) and 33\% had osteoporosis (75.1\% at LS, 9.3 at FN\%, and $15.6 \%$ at both sites). About $49.5 \%$ had calculated GFR(GFRc) $\geq 90$ (stage 1),39.2\% had GFRc of 60 through 89 (stage 2), and 11.3\% had GFRc of 30 through 59 (stage 3 ). The most prevalent cause of cirrhosis was hepatitis $\mathrm{C}$ (Table 1 ). There was no significant association between abnormal DEXA findings and the causes of cirrhosis. There was a correlation between T score in LS and FN (Spearman rho = 0.7; $\mathrm{P}<0.001$ ). Median of T score in LS was significantly less than that in FN $(\mathrm{P}<001)$. We found differences and similarities in association between variables and T scores of LS and FN (Table 2). Age had significant negative linear correlation only with T score of FN $(\mathrm{r}=-0.2 ; \mathrm{P}=0.02)$, but BMI, Child score, and GFRc had linear correlation with both LS and FN T score (Table 2).

The BMI of the patients with osteoporosis was significantly less than that of the patients with normal BMD ( $\mathrm{P}$ $<0.001)$ and osteopenia ( $\mathrm{P}<0.001)$. The Child score of patients with osteoporosis was significantly more than that of the patients with normal BMD $(\mathrm{P}=0.005)$ and osteopenia $(\mathrm{P}<0.005)$. Patients with osteoporosis had less GFRc than those with normal BMD did $(\mathrm{P}=0.001)$. Other variables including age, $25(\mathrm{OH})$ vitamin $\mathrm{D}, \mathrm{Ca}, \mathrm{P}, \mathrm{ALP}, \mathrm{PTH}$, and FBS showed no significant differences between the 
Hajiabbasi A et al.

groups (Table 3). The univariate analysis showed that the risk of abnormal DEXA findings (including both osteoporosis and/or osteopenia in all evaluated sites) did not significantly increased by age, sex, and lower vitamin D levels, but was significantly increased by lower BMI, current smoking, higher CHILD score, and lower GFRc (Table 4). After adjusting variables in a model with six variables including age, sex, BMI, CHILD score, current smoking, GFRc, and vitamin $\mathrm{D}$, we observed that lower vitamin $\mathrm{D}$, high CHILD score, and less GFRc values significantly increased the risk of abnormal DEXA findings (Table 5).

\begin{tabular}{ll}
\hline \multicolumn{2}{l}{ Table 1. Characteristics of Patients With Cirrhosis } \\
\hline Characteristics & Frequency, \% \\
\hline Age groups, $\mathbf{y}$ & \\
$\quad \leq 40$ & 19.6 \\
41 - 50 & 29.9 \\
51 - 60 & 23.7 \\
$\quad \geq 61$ & 26.8 \\
Male & 55.7 \\
Current smoker & 45.4 \\
Cause of cirrhosis & \\
\hline Hepatitis B & 26.8 \\
\hline Hepatitis C & 40.2 \\
\hline Cryptogenic & 21.6 \\
\hline Others ${ }^{\text {a }}$ & 11.4 \\
\hline Child groups & 16.5 \\
\hline A & 47.4 \\
\hline B & 36.1 \\
\hline C \\
\hline ancluding, alcohol-induced cirrhosis, autoimmune cirrhosis, primary \\
biliary cirrhosis, and cirrhotic patient due to Budd-Chiari syndrome or \\
sclerosing cholangitis.
\end{tabular}

Table 2. Linear Correlation of Variables With T Score in Two Different Anatomical Sites ${ }^{\text {a }}$

\begin{tabular}{|c|c|c|}
\hline Variables & $\begin{array}{c}\text { T score of LS; } \mathbf{r} \\
\text { (P value) }\end{array}$ & $\begin{array}{l}\text { score of FN; r (P } \\
\text { value) }\end{array}$ \\
\hline Age $^{\mathrm{b}}$ & $-0.03(\mathrm{NS})$ & $-0.2(0.02)$ \\
\hline BMI $^{\mathrm{C}}$ & $0.5(<0.001)$ & $0.4(<0.001)$ \\
\hline Child score $^{\mathrm{C}}$ & $-0.5(<0.001)$ & $-0.3(0.003)$ \\
\hline Low $25(\mathrm{OH})$ vitamin $\mathrm{D}^{\mathrm{C}}$ & $-0.02(\mathrm{NS})$ & $-0.2(\mathrm{NS})$ \\
\hline $\mathbf{A L P}^{\mathrm{C}}$ & $-0.1(\mathrm{NS})$ & $-0.04(\mathrm{NS})$ \\
\hline $\mathbf{P T H}^{\mathrm{C}}$ & $-0.4(\mathrm{NS})$ & $-0.2(\mathrm{NS})$ \\
\hline $\mathrm{Ca}^{\mathrm{C}}$ & $0.2(\mathrm{NS})$ & $0.1(\mathrm{NS})$ \\
\hline $\mathbf{P}^{\mathrm{C}}$ & $-0.06(\mathrm{NS})$ & 0.05 (NS) \\
\hline BUN $^{\mathrm{C}}$ & $-0.1(\mathrm{NS})$ & $-0.2(\mathrm{NS})$ \\
\hline $\mathrm{Cr}^{\mathrm{C}}$ & $-0.1(\mathrm{NS})$ & $-0.1(\mathrm{NS})$ \\
\hline GFRc $^{\mathrm{C}}$ & $0.3(0.01)$ & $0.3(0.002)$ \\
\hline FBS $^{\mathrm{C}}$ & $-0.1(\mathrm{NS})$ & $-0.1(\mathrm{NS})$ \\
\hline \multicolumn{3}{|c|}{$\begin{array}{l}\text { a Abbreviations: LS, lumbar spine; FN, femoral neck 3; NS, not } \\
\text { significant; ALP, alkaline phosphatase, PTH, parathyroid hormone; Ca, } \\
\text { calcium; P, phosphorus; BUN, blood urea nitrogen; Cr, creatinine, FBS, } \\
\text { fasting blood sugar; and GFRc, calculated glomerular filtration rate. } \\
\text { b Correlation coefficient (r). } \\
\text { c Rho spearman. }\end{array}$} \\
\hline
\end{tabular}

Table 3. Comparing Variables in Patients with Diagnosis of Normal Bone Mineral Density, Osteopenia, and Osteoporosis a,b

\begin{tabular}{lccc}
\hline Variables & $\begin{array}{c}\text { Normal Versus; } \\
\text { Osteopenia }\end{array}$ & $\begin{array}{c}\text { Normal Versus } \\
\text { Osteoporosis }\end{array}$ & $\begin{array}{c}\text { Osteoporosis Versus } \\
\text { Osteopenia }\end{array}$ \\
\hline Age & NS & NS & NS \\
BMI & NS & P $<0.001$ & P $<0.001$ \\
Child value & NS & P $<0.001$ & 0.005 \\
Ca & NS & NS & NS \\
P & NS & NS & NS \\
PTH & NS & NS & NS \\
ALP & NS & NS & NS \\
Vitamin D & NS & NS & NS \\
Cr & NS & NS & NS \\
GFRc & NS & 0.001 & NS \\
FBS & NS & NS & NS \\
\hline
\end{tabular}

a Abbreviations: NS, not significant; Ca, calcium; P, phosphorus; PTH, parathyroid hormone; ALP, alkaline phosphatase; $\mathrm{Cr}$, creatinine; GFRc, calculated glomerular filtration rate; and FBS, fasting blood sugar. b $\alpha$ corrected $<0.01$.

Table 4. Variables Associated With Abnormal Dual-Energy X-Ray Absorptiometry Findings (Univariate Analysis) ${ }^{a}$

\begin{tabular}{lccc}
\hline Variable & OR & $\mathbf{9 5 \%}$ CI & P Value \\
\hline Age & 1.02 & $0.98-1.06$ & NS \\
Gender & 0.5 & $0.2-1.4$ & NS \\
BMI & 0.8 & $0.7-0.98$ & 0.03 \\
Child score & 1.5 & $1.1-2.0$ & 0.004 \\
Current smoking & 0.3 & $0.01-0.89$ & 0.03 \\
GFRc & 0.97 & $0.96-0.99$ & 0.005 \\
Vitamin D & 0.97 & $0.93-1.01$ & NS \\
\hline
\end{tabular}

a Abbreviations: GFR, calculated glomerular filtration rate; NS, not significant.

Table 5. Variables Associated With Abnormal DEXA Findings (Multivariate Analysis) $^{\mathrm{a}}$

\begin{tabular}{lccc}
\hline Variable & OR & 95\% CI & P Value \\
\hline CHILD score & 1.7 & $1.2-2.5$ & 0.004 \\
GFRc & 0.96 & $0.93-0.99$ & 0.01 \\
Vitamin D & 0.95 & $0.90-0.99$ & 0.04 \\
\hline
\end{tabular}

a Abbreviation: GFRc, calculated glomerular filtration rate.

\section{Discussion}

Determinants of BMD such as genetic factors (the main determinant of peak bone mass), sex, age, BMI, lifestyle such as sun exposure, physical activity, smoking, and alcohol consumption, food habits such as salt, protein, and dairy consumption, use of some medications and prevalence of many diseases such as cirrhosis are not similar in different regions of the world. In Iran, $72.1 \%$ of men and 
$75.1 \%$ of women have vitamin D deficiency (11), the mean of salt consumption is high (12), 35.7\% are physically inactive (13), and $11.8 \%$ are current smokers $(22.1 \%$ of men and $1.3 \%$ of women) (14). In our study, $78.4 \%$ of patients with cirrhosis had abnormal DEXA findings. In general, among Iranian population, the prevalence rates of osteopenia and osteoporosis in LS are respectively 35\% (95\% CI, $30 \%-39 \%$ ) and $17 \%$ (95\% CI, 13\% - 20\%) (15). Our study showed that the prevalence of both osteopenia (45.4\%) and osteoporosis $(29.9 \%)$ were higher in patients with cirrhosis than in normal population. In patients with cirrhosis the prevalence of osteopenia and osteoporosis has been heterogeneous in different reports, ranging from $11.5 \%$ to $48.1 \%$ for osteopenia and from $1.9 \%$ to $36.6 \%$ for osteoporosis $(6,7,16-19)$. This heterogeneity can be attributable to the different methodology of the studies, patients' characteristics definition of osteopenia and osteoporosis ( $\mathrm{T}$ or Z score), etiologies of cirrhosis, its severity, and factors related to the different parts such as lifestyle and food habits. In Spain, in a descriptive cross-sectional study on 489 patients with cirrhosis (male $79 \%$, mean age of 53 years), Vargas et al. (19) showed that the prevalence of abnormal bone density is $72 \%$. In that study, $59 \%$ had alcoholic cirrhosis and 78\% had decompensated cirrhosis. In our study, prevalence of alcoholic cirrhosis was much lower, but the rate of decompensated cirrhosis was higher than that of Spanish study. In Turkey, Goral et al. (7) showed that the prevalence of osteoporosis in a study on 55 patients with cirrhosis (males, 69\%; mean age, 44.8 years) was $37 \%$. Hepatitis C and primary biliary cirrhosis constituted $2 \%$ and $3.6 \%$ of etiologies of cirrhosis, respectively. In this study, $41 \%$ of the patients had decompensated cirrhosis that was significantly lower than the rate in our study. This may demonstrate the prevalence difference. In another Turkish study on 44 males with cirrhosis (mean age, 50.8 years), Soylu et al. (6) showed that $20 \%$ of the patients had osteopenia and $1.9 \%$ had osteoporosis, much lower than the rates in our study. In another crosssectional study in the United Kingdom, Ninkovic et al. (17) showed that the prevalence rates of osteopenia and osteoporosis in patients with cirrhosis were $48.8 \%$ and $36.6 \%$, respectively. The characteristics of participants in that study were as follows: mean age, 51.1 years; males, 57.7\%; and decompensated cirrhosis, 95\%. Etiologies of cirrhosis were alcohol consumption in $18.9 \%$, hepatitis B in $7.4 \%$ and primary biliary cirrhosis in $20.6 \%$. Decompensated cirrhosis was reported to be $95 \%$ and the etiologies of cirrhosis were significantly different from our study. In a cross-sectional study on 104 patients with cirrhosis who were awaiting for liver transplantation in the United States (males, 51.9\%; mean age, 54.4 years), Sokhi et al. (16) demonstrated that $34.6 \%$ had osteopenia and $11.5 \%$ had osteoporosis. The etiologies of cirrhosis were alcohol consumption (16.3\%), hepatitis C (66.4\%), cholestatic diseases (7.7\%), and other causes (9.6\%).

Patients who were awaiting for liver transplantation take more care than the others do. In addition, at the time of this study, all patients were receiving daily multivitamins that included $0.25 \mu \mathrm{g}$ of vitamin D that might influence the results of bone densitometry. Imbalance in bone resorption and formation varies at different skeletal sites. Bone loss occurs due to both or one of these mechanisms: first, an increase in osteoclastic activity and/or a decrease in osteoblastic activity; and second, increase in the number of remodeling sites (20). Based on our findings, the role of variables in inducing abnormal BMD might be related to the severity of bone loss and its anatomical sites. Researchers in a hybrid of two casecontrol interview-based study had shown that the role of factors in protection against or inducing osteoporosis in LS could be different from that in FN region. Our study showed that abnormal DEXA was related to severity of cirrhosis but not to the etiology of cirrhosis. Our study showed that the degree of bone loss was related to severity of cirrhosis and not the etiology of chronic liver disease. These are similar to findings of Giouleme et al. (21) study on 83 hospitalized patients with cirrhosis (males, $61.4 \%$; median age of men, 59 years; median age of women, 57 years). The etiologies of cirrhosis were viral hepatitis (49 cases), alcohol (16 cases), primary biliary cirrhosis (1 case), steatohepatitis (1 case), autoimmune hepatitis (2 cases), Wilson disease (1 case), and unknown etiologies (7 cases). Overall, $40.9 \%$ of them had decompensated cirrhosis. Some potential inciting factors that either directly or indirectly alter bone mass, i.e. insulin growth factor-1 (IGF-1) deficiency, hyperbilirubinemia, and hypogonadism (estrogen and testosterone deficiency), were commonly seen in cirrhosis by any causes (22). Depression of osteoblastic function may be related to jaundice, independent of etiology (23). Some other inciting factors such as alcoholism, excess tissue iron deposition, and corticosteroid therapy, which could be seen in specific causes of cirrhosis, were infrequently reported in our patients. The effect of etiology on abnormal BMD could be evaluated by conducting a meta-analysis on similar studies, which mentioned different etiologies of chronic liver diseases. In present study, higher Child score and lower vitamin D levels were found to be risk factors for abnormal DEXA findings in patients with cirrhosis. Serum levels of 25 $(\mathrm{OH})$ vitamin D in patients with chronic liver disease were decreased and this level would be reduced more with the progression of cirrhosis (24). It seems that both reduced exposure to UV light and dietary insufficiency account for vitamin D deficiency in the majority of cases. There is also impaired cutaneous synthesis of vitamin D in the presence of jaundice (24). As vitamin D insufficiency is associated with secondary hyperparathyroidism, increased bone turnover, and accelerated bone loss, administration of vitamin D supplementation seems to be a reasonable approach in these patients. Lower GFR was another risk factor for abnormal BMD in our study. Activation of 25 $(\mathrm{OH})$ vitamin D in kidney needs a GFR $\geq 30 \mathrm{~mL} / \mathrm{min}$; thus, simultaneous measurement of $25(\mathrm{OH})$ vitamin $\mathrm{D}$ and 1 , $25(\mathrm{OH})$ vitamin D must be considered for more precise 
detection of the role of vitamin D in lowering BMD (24, 25). In patients with cirrhosis, GFR overestimates renal function (20\% - 400\%); thus, GFR could not estimate real renal function $(24,26-28)$. This should be mentioned as another important point. In addition, for confirmation of the association between GFR and abnormal DEXA findings in future studies, we suggest direct measurement of renal function by administration of a radioactive isotope (such as inulin, iothalamate), which was not available in our setting.

Our study had some limitations including unknown etiology in some of our cases with cirrhosis, drugs that could not be discontinued, technically overestimation of GFR, and high prevalence of vitamin D insufficiency in our region and the lack of measurement of (1.25) OH Vitamin D.

In conclusion, metabolic bone disease is common among patients with chronic liver disease; osteoporosis and osteopenia account for the majority of cases. Regardless of the etiology of cirrhosis, bone disease in these patients may cause an increased incidence of bone pain and fractures, a major source of morbidity before and after liver transplantation. This study demonstrated that abnormal BMD (osteopenia and/or osteoporosis) is highly prevalent among patients with cirrhosis (78.4\%). Higher Child score, vitamin D deficiency, and lower GFR correlate with abnormal BMD of both LS and FN. According to our findings, we recommend correction of vitamin $\mathrm{D}$ level in chronic liver disease, especially in patients with cirrhosis to maintain their bone density. We should pay more attention to renal function of this group of patients. These important cares should be emphasized in higher Child score, ie, in patients with decompensated cirrhosis.

\section{Acknowledgements}

We would like to thank Dr. Siamak Geranmayeh and Dr. Parvin Sattari for their helps in conducting this study.

\section{Authors' Contributions}

Study concept and design: Asghar Hajiabbasi, Afshin Shafaghi, and Alireza Amir Maafi; Acquisition of data: Alireza Amir Maafi, Haniyeh Sadat Fayazi, and Pooneh Ghavidel Parsa; Analysis and interpretation of data: Mohammad Hassan Hedayati Emami and Irandokht Shenavar Masooleh; Drafting of the manuscript: Asghar Hajiabbasi, Afshin Shafaghi, and Alireza Amir Maafi; Critical revision of the manuscript for important intellectual content: Irandokht Shenavar Masooleh and Mohammad Hassan Hedayati Emami; Statistical analysis: Afshin Shafaghi and Pooneh Ghavidel Parsa; Administrative, technical, and material support: Pooneh Ghavidel Parsa; Study supervision: Asghar Hajiabbasi and Afshin Shafaghi.

\section{Funding/Support}

This study was supported by Guilan University of Medical Sciences, Rasht, IR Iran.

\section{References}

1. Felecia C, David D. Rheumatology. Mosbey: Elsevier; 2011. Pathogenesis of osteoporosis.Pathogenesis of osteoporosis.

2. Lane NE. Metabolic Bone Disease. In: Firestein GS, Budd RC, Gabriel EG, Mcinnes IB, O'dell JR editors. Kelley's Textbook of Rheumatology.; 2013. pp. 1660-79.

3. Bringhust FR, Demay MB, Krane SM, Konenberg HM. Bone and Mineral Metabolism in Health and Disease. In: Longo DL, Fauci AS, Kasper DL, Hauser SL, Jameson JL, Loscalzo J editors. Harrison's Principles of Internal Medicine. 18th ed; 2012. pp. 3082-95.

4. Luxon BA. Bone disorders in chronic liver diseases. Curr Gastroenterol Rep. 2011;13(1):40-8.

5. Guanabens N, Pares A. Liver and bone. Arch Biochem Biophys. 2010;503(1):84-94.

6. Soylu AR, Tuglu C, Arikan E, Yetisyigit T, Kunduracılar H, Koker $\mathrm{IH}$, et al. The Role of Serum Cytokines in the Pathogenesis of Hepatic Osteodystrophy in Male Cirrhotic Patients. Gastroenterol Res Pract. 2012;2012.

7. Goral V, Simsek M, Mete N. Hepatic osteodystrophy and liver cirrhosis. World J Gastroenterol. 2010;16(13):1639-43.

8. Collier JD, Ninkovic M, Compston JE. Guidelines on the management of osteoporosis associated with chronic liver disease. Gut. 2002;50 Suppl 1:i1-9.

9. Lewiecki EM, Rosen CJ. Overview of dual-energy x-ray absorptiometry: UpToDate Marketing Professional; 2013. Available from: http://www.uptodate.com/contents/overview-of-dual-energy-xray-absorptiometry? source=search_result\&search=DXA\&select edTitle $=1 \sim 116$.

10. Ghany MG, Hoofnagle JH. Approach to the patient with liver disease. In: Longo DL, Fauci AS, Hauser SL, Jameson JL, Loscalzo J editors. Harrison's Principles of Internal Medicine. 18th ed; 2012. pp. 2520-6.

11. Moradzadeh K, Larijani B, Keshtkar AA, Hossein-Nezhad A, Rajabian R, Nabipour I, et al. Normative values of vitamin D among Iranian population: a population based study. In J Osteoporos Metab Disord . 2008;1(1):8-15.

12. Fahimi S, Pharoah P. Reducing salt intake in Iran: priorities and challenges. Arch Iran Med. 2012;15(2):110-2.

13. World Health Organization.. Country profile Iran. 2011. Available from: http://www.who.int/nmh/countries/2011/irn_en.pdf.

14. World Health Organization.. WHO Report on the Global Tobacco Epidemic, 2013. Country profile. Iran (Islamic Republic of). 2013. Available from: http://www.who.int/tobacco/surveillance/policy/ country_profile/irn.pdf?ua=1.

15. Irani AD, Poorolajal J, Khalilian A, Esmailnasab N, Cheraghi Z. Prevalence of osteoporosis in Iran: A meta-analysis. J Res Med Sci. 2013;18(9):759-66.

16. Sokhi RP, Anantharaju A, Kondaveeti R, Creech SD, Islam KK, Van Thiel DH. Bone mineral density among cirrhotic patients awaiting liver transplantation. Liver Transpl. 2004;10(5):648-53.

17. Ninkovic M, Love SA, Tom B, Alexander GJ, Compston JE. High prevalence of osteoporosis in patients with chronic liver disease prior to liver transplantation. Calcif Tissue Int. 2001;69(6):321-6.

18. Loria I, Albanese C, Giusto M, Galtieri PA, Giannelli V, Lucidi C, et al. Bone disorders in patients with chronic liver disease awaiting liver transplantation. Transplant Proc. 2010;42(4):1191-3.

19. Alcalde Vargas A, Pascasio Acevedo JM, Gutierrez Domingo I, Garcia Jimenez R, Sousa Martin JM, Ferrer Rios MT, et al. Prevalence and characteristics of bone disease in cirrhotic patients under evaluation for liver transplantation. Transplant Proc. 2012;44(6):1496-8.

20. lindsay R, Cosman F. Osteoporosis. In: Longo DL, Fauci AS, Kasper LD, Hauser SL, Jameson JL editors. Harrison's Principles of Internal Medicine.18th ed; 2012. pp. 3120-35.

21. Giouleme OI, Vyzantiadis TA, Nikolaidis NL, Vasiliadis TG, Papageorgiou AA, Eugenidis NP, et al. Pathogenesis of osteoporosis in liver cirrhosis. Hepatogastroenterology. 2006;53(72):938-43.

22. Rouillard S, Lane NE. Hepatic osteodystrophy. Hepatology. 2001;33(1):301-7.

23. Janes CH, Dickson ER, Okazaki R, Bonde S, McDonagh AF, Riggs BL. Role of hyperbilirubinemia in the impairment of osteoblast proliferation associated with cholestatic jaundice. J Clin Invest. 
1995;95(6):2581-6.

24. Francoz C, Glotz D, Moreau R, Durand F. The evaluation of renal function and disease in patients with cirrhosis. $J$ Hepatol. 2010;52(4):605-13.

25. Teneva BH. Pathogenesis and assessment of renal function in patients with liver cirrhosis. Folia Med (Plovdiv). 2012;54(4):5-13.

26. Sherman DS, Fish DN, Teitelbaum I. Assessing renal function in cirrhotic patients: problems and pitfalls. Am J Kidney Dis 2003;41(2):269-78.

27. Papadakis MA, Arieff AI. Unpredictability of clinical evaluation of renal function in cirrhosis. Prospective study. Am J Med. 1987;82(5):945-52

28. Gines P, Guevara M, Arroyo V, Rodes J. Hepatorenal syndrome. Lancet. 2003;362(9398):1819-27. 